Supplementary information associated with

\title{
HILIC-MRM-MS for linkage-specific separation of sialylated glycopeptides to quantify Prostate-Specific Antigen Proteoforms
}

\author{
Yuri van der Burgt, Kasper M. Siliakus, Christa M. Cobbaert, L. Renee Ruhaak
}

Department of Clinical Chemistry and Laboratory Medicine, Leiden University Medical Center, Albinusdreef 22333 ZA Leiden, The Netherlands

\section{Table of Content}

1. Supplementary Table S1. Transitions developed for tryptic peptides and glycopeptides from PSA

2. Supplementary Table S2. Transitions developed for ArgC peptides and glycopeptides from PSA

3. Supplementary Figure S1. Optimization of digestion conditions

4. Supplementary Figure S2. Repeatability of the LC-MRM-MS analysis of PSA 
Tables

Supplementary Table S1. Transitions developed for tryptic peptides and glycopeptides from PSA.

\begin{tabular}{|c|c|c|c|c|c|c|c|c|c|}
\hline \multirow[b]{2}{*}{ AA } & \multirow[b]{2}{*}{ peptide } & \multirow{2}{*}{$\begin{array}{l}\mathrm{RT} \\
\mathrm{min}\end{array}$} & \multirow{2}{*}{$\begin{array}{c}\text { Precursor } \\
\mathrm{m} / \mathrm{z}\end{array}$} & \multicolumn{2}{|c|}{ Quantifier } & \multicolumn{2}{|c|}{ Qualifier 1 } & \multicolumn{2}{|c|}{ Qualifier 2} \\
\hline & & & & $\mathrm{m} / \mathrm{z}$ & $\mathrm{CE}$ & $\mathrm{m} / \mathrm{z}$ & $\mathrm{CE}$ & $\mathrm{m} / \mathrm{z}$ & $\mathrm{CE}$ \\
\hline $25-33$ & IVGGWECEK & 3.4 & $539.2(2+)$ & $865.4(\mathrm{y} 7,1+)$ & 15 & $964.4(\mathrm{y} 8,1+)$ & 18 & $213.2(\mathrm{~b} 2,1+)$ & 15 \\
\hline $110-125$ & FLRPGDDSSHDLMLLR & 3.2 & $468.7(4+)$ & $532.3(\mathrm{y} 4,1+)$ & 15 & $760.4(\mathrm{y} 6,1+)$ & 18 & $645.4(\mathrm{y} 5,1+)$ & 18 \\
\hline $126-137$ & $\begin{array}{l}\text { LSEPAELTDAVK } \\
\text { SIL- }\end{array}$ & 3.6 & $636.8(2+)$ & $943.5(\mathrm{y} 9,1+)$ & 18 & $1072.6(\mathrm{y} 10,1+)$ & 20 & $201.2(\mathrm{~b} 2,1+)$ & 22 \\
\hline- & FLRPGDDSSHDLMLLR & 3.2 & $471.2(4+)$ & $542.3(\mathrm{y} 4,1+)$ & 15 & $770.4(y 6,1+)$ & 18 & $655.4(\mathrm{y} 5,1+)$ & 18 \\
\hline- & SIL-LSEPAELTDAVK & 3.6 & $640.8(2+)$ & $951.5(\mathrm{y} 9,1+)$ & 18 & $1080.6(\mathrm{y} 10,1+)$ & 20 & $201.2(\mathrm{~b} 2,1+)$ & 22 \\
\hline $69-70$ & NK-H5N4F1S2 & $6.2,6.3,6.4$ & $871.3(3+)$ & $204.0(1+)$ & 30 & $274.0(1+)$ & 30 & - & - \\
\hline $69-70$ & NK-H5N4F0S2 & & $822.6(3+)$ & $204.0(1+)$ & 30 & $274.0(1+)$ & 30 & - & - \\
\hline $69-70$ & NK-H4N5F1S2 & & $885.0(3+)$ & $204.0(1+)$ & 30 & $274.0(1+)$ & 30 & - & - \\
\hline $69-70$ & NK-H4N5F0S2 & & $836.3(3+)$ & $204.0(1+)$ & 30 & $274.0(1+)$ & 30 & - & - \\
\hline $69-70$ & NK-H3N6F1S2 & & $898.7(3+)$ & $204.0(1+)$ & 30 & $274.0(1+)$ & 30 & - & - \\
\hline $69-70$ & NK-H5N4F1S1 & & $774.3(2+)$ & $204.0(1+)$ & 30 & $274.0(1+)$ & 30 & - & - \\
\hline $69-70$ & NK-H4N3F1S1 & & $978.4(2+)$ & $204.0(1+)$ & 30 & $274.0(1+)$ & 30 & - & - \\
\hline $69-70$ & NK-H4N3F0S1 & & $905.4(2+)$ & $204.0(1+)$ & 30 & $274.0(1+)$ & 30 & - & - \\
\hline $69-70$ & NK-H3N4F1S1 & & $998.9(2+)$ & $204.0(1+)$ & 30 & $274.0(1+)$ & 30 & - & - \\
\hline $69-70$ & NK-H5N4F1S0 & & $1015.4(2+)$ & $204.0(1+)$ & 30 & $366.0(1+)$ & 30 & - & - \\
\hline $69-70$ & NK-H4N3F1S0 & & $832.8(2+)$ & $204.0(1+)$ & 30 & $366.0(1+)$ & 30 & - & - \\
\hline $69-70$ & NK-H4N4F1S0 & & $934.4(2+)$ & $204.0(1+)$ & 30 & $366.0(1+)$ & 30 & - & - \\
\hline $69-70$ & NK-H4N5F1S0 & & $1035.9(2+)$ & $204.0(1+)$ & 30 & $366.0(1+)$ & 30 & - & - \\
\hline
\end{tabular}


Supplementary Table S2. Transitions developed for ArgC peptides and glycopeptides from PSA

\begin{tabular}{|c|c|c|c|c|c|c|c|c|}
\hline \multirow[t]{2}{*}{$\mathrm{AA}$} & \multirow[t]{2}{*}{ peptide } & \multirow{2}{*}{$\begin{array}{c}\text { Precursor } \\
\mathrm{m} / \mathrm{z}\end{array}$} & \multicolumn{2}{|c|}{ Quantifier } & \multicolumn{2}{|c|}{ Qualifier 1} & \multicolumn{2}{|c|}{ Qualifier 2} \\
\hline & & & $\mathrm{m} / \mathrm{z}$ & $\mathrm{CE}$ & $\mathrm{m} / \mathrm{z}$ & $\mathrm{CE}$ & $\mathrm{m} / \mathrm{z}$ & $\mathrm{CE}$ \\
\hline $48-68$ & AVCGGVLVHPQWVLTAAHCIR & $586.8(4+)$ & $544.3(\mathrm{~b} 6,1+)$ & 12 & $941.5(\mathrm{y} 8,1+)$ & 25 & $726.4(\mathrm{y} 6,1+)$ & 15 \\
\hline $110-125$ & FLRPGDDSSHDLMLLR & $468.7(4+)$ & $532.3(\mathrm{y} 4,1+)$ & 15 & $760.4(\mathrm{y} 6,1+)$ & 18 & $645.4(\mathrm{y} 5,1+)$ & 18 \\
\hline $251-261$ & KWIKDTIVANP & $428.9(3+)$ & $116.1(\mathrm{y} 1,1+)$ & 10 & $1055.6(\mathrm{~b} 9,1+)$ & 12 & $885.5(\mathrm{~b} 7,1+)$ & 15 \\
\hline - & SIL-FLRPGDDSSHDLMLLR & $471.2(4+)$ & $542.3(\mathrm{y} 4,1+)$ & 15 & $770.4(\mathrm{y} 6,1+)$ & 18 & $655.4(\mathrm{y} 5,1+)$ & 18 \\
\hline- & SIL-KWIKDTIVANP & $430.9(3+)$ & $116.1(\mathrm{y} 1,1+)$ & 10 & $1061.6(\mathrm{~b} 9,1+)$ & 12 & $885.5(\mathrm{~b} 7,1+)$ & 15 \\
\hline $69-77$ & NKSVILLGR-H5N4F1S2 & 1117.5 & $204.0(1+)$ & 30 & $274.0(1+)$ & 30 & - & - \\
\hline $69-77$ & NKSVILLGR-H5N4F0S2 & 1068.8 & $204.0(1+)$ & 30 & $274.0(1+)$ & 30 & - & - \\
\hline $69-77$ & NKSVILLGR-H4N5F1S2 & 1131.2 & $204.0(1+)$ & 30 & $274.0(1+)$ & 30 & - & - \\
\hline $69-77$ & NKSVILLGR-H4N5F0S2 & 1082.5 & $204.0(1+)$ & 30 & $274.0(1+)$ & 30 & - & - \\
\hline $69-77$ & NKSVILLGR-H3N6F1S2 & 1144.8 & $204.0(1+)$ & 30 & $274.0(1+)$ & 30 & - & - \\
\hline $69-77$ & NKSVILLGR-H5N4F1S1 & 1020.5 & $204.0(1+)$ & 30 & $274.0(1+)$ & 30 & - & - \\
\hline $69-77$ & NKSVILLGR-H4N3F1S1 & 898.7 & $204.0(1+)$ & 30 & $274.0(1+)$ & 30 & - & - \\
\hline $69-77$ & NKSVILLGR-H4N3F0S1 & 850.1 & $204.0(1+)$ & 30 & $274.0(1+)$ & 30 & - & - \\
\hline $69-77$ & NKSVILLGR-H3N4F1S1 & 912.4 & $204.0(1+)$ & 30 & $274.0(1+)$ & 30 & - & - \\
\hline $69-77$ & NKSVILLGR-H5N4F1S0 & 923.4 & $204.0(1+)$ & 30 & $366.0(1+)$ & 30 & - & - \\
\hline $69-77$ & NKSVILLGR-H4N3F1S0 & 801.7 & $204.0(1+)$ & 30 & $366.0(1+)$ & 30 & - & - \\
\hline $69-77$ & NKSVILLGR-H4N4F1S0 & 869.4 & $204.0(1+)$ & 30 & $366.0(1+)$ & 30 & - & - \\
\hline $69-77$ & NKSVILLGR-H4N5F1S0 & 937.1 & $204.0(1+)$ & 30 & $366.0(1+)$ & 30 & - & - \\
\hline
\end{tabular}




\begin{tabular}{|l|c|c|c|c|}
\hline Condition & Buffer & $\mathbf{C a C l} 2$ & EDTA & ArgC \\
\hline 1 & $50 \mathrm{mM}$ TRIS & $50 \mathrm{mM}$ & $50 \mathrm{mM}$ & $1: 35$ \\
\hline 2 & $50 \mathrm{mM}$ TRIS & $50 \mathrm{mM}$ & $50 \mathrm{mM}$ & $1: 20$ \\
\hline 3 & $50 \mathrm{mM}$ TRIS & $50 \mathrm{mM}$ & $50 \mathrm{mM}$ & $1: 10$ \\
\hline 4 & $50 \mathrm{mM}$ TRIS & $50 \mathrm{mM}$ & $50 \mathrm{mM}$ & $1: 5$ \\
\hline 5 & $50 \mathrm{mM}$ TRIS & $50 \mathrm{mM}$ & $0 \mathrm{mM}$ & $1: 35$ \\
\hline 6 & $50 \mathrm{mM}$ TRIS & $0 \mathrm{mM}$ & $50 \mathrm{mM}$ & $1: 35$ \\
\hline 7 & $50 \mathrm{mM}$ TRIS & $0 \mathrm{mM}$ & $0 \mathrm{mM}$ & $1: 35$ \\
\hline 8 & $50 \mathrm{mM}$ TRIS & $5 \mathrm{mM}$ & $0 \mathrm{mM}$ & $1: 35$ \\
\hline 9 & $50 \mathrm{mM}$ TRIS & $20 \mathrm{mM}$ & $0 \mathrm{mM}$ & $1: 35$ \\
\hline 10 & $10 \mathrm{mM}$ TRIS & $0 \mathrm{mM}$ & $0 \mathrm{mM}$ & $1: 35$ \\
\hline 11 & $100 \mathrm{mM}$ TRIS & $0 \mathrm{mM}$ & $0 \mathrm{mM}$ & $1: 35$ \\
\hline 12 & $50 \mathrm{mM} \mathrm{ABC}$ & $5 \mathrm{mM}$ & $0 \mathrm{mM}$ & $1: 35$ \\
\hline 13 & $50 \mathrm{mM}$ ABC & $50 \mathrm{mM}$ & $0 \mathrm{mM}$ & $1: 35$ \\
\hline
\end{tabular}

FLRPGDDSSHDLMLLR

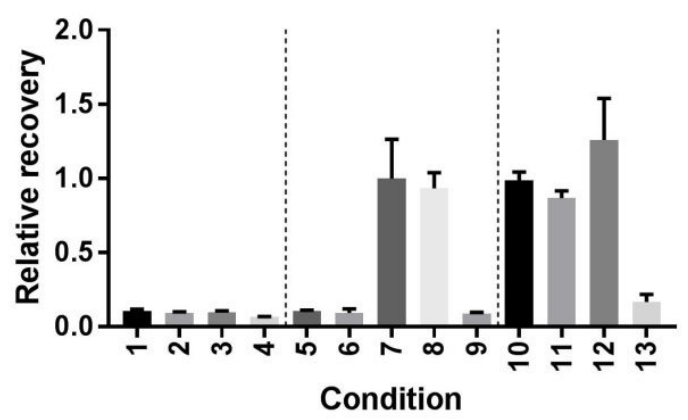

KWIKDTIVANP

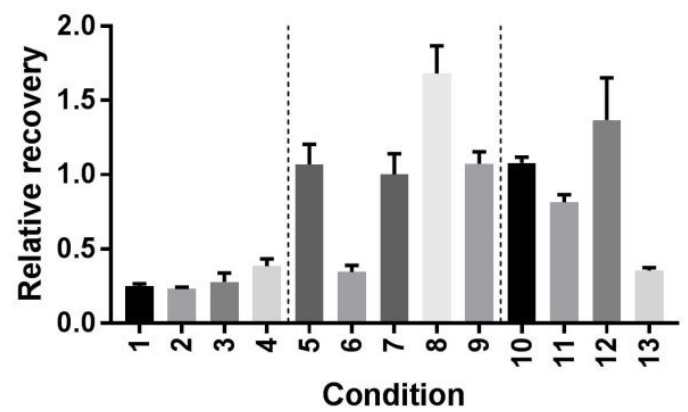

AVCGGVLVHPQWVLTAAHCIR

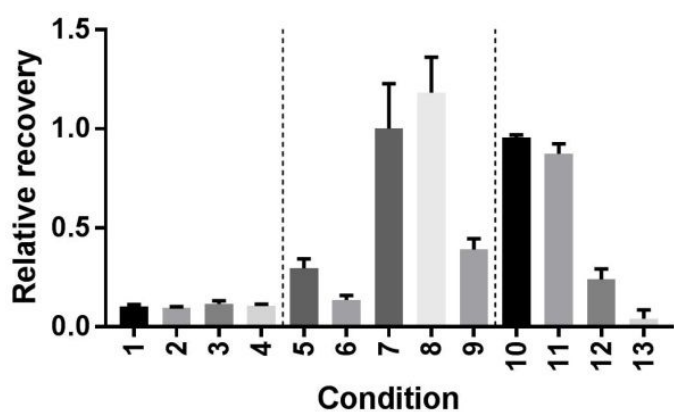

NKSVILLGR-H5N4F1S2

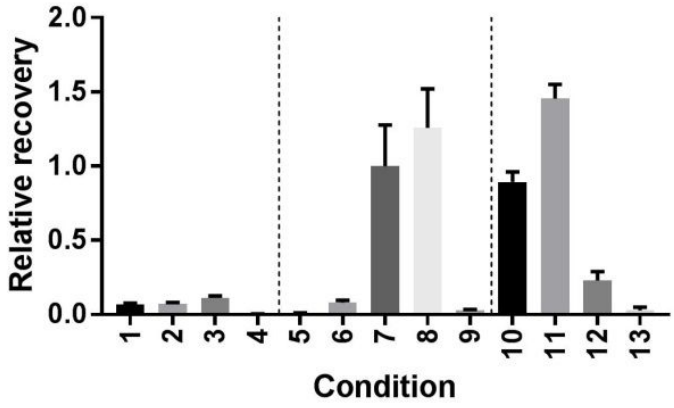

Supplementary Figure S1. Optimization of digestion conditions. 


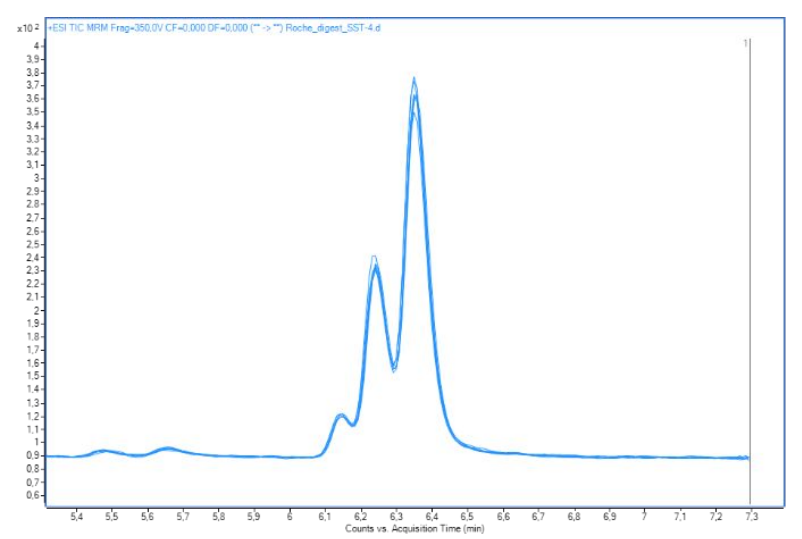

Supplementary Figure S2. Overlay of seven analyses of a PSA standard digest (for experimental details, see the main publication, experimental section). These digests were measured over a $48 \mathrm{~h}$. time interval. The injection volume was reduced to $1 \mu \mathrm{L}$ to allow for a larger number of injections from the same vial. These results indicate the repeatability of the LC-MRM-MS measurement both in terms of quantitation as well as retention time. 